\title{
Internal Jugular Vein Ultrasonography in Prediction of Postspinal Hypotension
}

\author{
Yeliz Kılıç, Sema Sanal Bas, Gülay Erdoğan Kayhan and Mehmet Sacit Güleç \\ Department of Anesthesiology and Reanimation, Faculty of Medicine, Osmangazi University, Eskişehir, Turkey
}

\begin{abstract}
Objective: To determine the role of internal jugular vein (IJV) ultrasonography to predict postspinal hypotension in patients undergoing elective surgery.

Study Design: A prospective observational study.

Place and Duration of Study: Department of Anesthesiology and Reanimation, Osmangazi University, Eskişehir, Turkey, from January to September 2020.

Methodology: Patients aged between 18 and 65 years, who underwent elective surgery under spinal anesthesia, were included in the study. Sonographic examination of IJV was performed before spinal anesthesia. Significant postspinal hypotension was defined as a mean arterial pressure less than $65 \mathrm{mmHg}$ or more than $20 \%$ decrease compared to the baseline value. The patients were classified as hypotensive and non-hypotensive groups. The association between sonographic IJV measurements and postspinal hypotension was analysed.

Results: The evaluation was performed on 47 patients. Twenty-two (46.8\%) patients developed postspinal hypotension. The two groups were similar in all baseline characteristics $(p>0.05)$. Among all sonographic measurements, IJV collapsibility index was significantly different between the two groups $(p=0.014)$. Receiver operating characteristic curve analysis showed that IJV collapsibility index had a sensitivity of $64 \%$ and a specificity of $63.6 \%$ to predict the postspinal hypotension at a cut-off point of $22.6 \%$. Area under curve (AUC) was 0.709 .

Conclusion: Despite the moderate sensitivity and specifity rates, IJV collapsibility index can be considered as an alternative predictor of postspinal hypotension.
\end{abstract}

Key Words: Internal jugular vein, Postspinal hypotension, Spinal anesthesia, Ultrasonography.

How to cite this article: Kılıç Y, Bas SS, Kayhan GE, Güleç MS. Internal Jugular Vein Ultrasonography in Prediction of Postspinal Hypotension. J Coll Physicians Surg Pak 2020; 30(12):1256-1261.

\section{INTRODUCTION}

Hypotension after spinal anesthesia is a common event in routine anesthesia practice, with a reported incidence of up to $90 \% .{ }^{1}$ Although advanced age and sensory block above fifth thoracic vertebrae have been reported as the most important causes of postspinal hypotension, the presence of low intravascular volume related to chronic systemic diseases, poor physical condition, medications, and long fasting time is also a well known predisposing factor for this complication. ${ }^{2,3}$ Intraoperative hypotension, if severe or prolonged, is associated with the development of serious conditions such as stroke, acute myocardial infarction, heartand kidney failure, prolonged hospitalization, and increased risk of postoperative mortality. ${ }^{4-6}$

Correspondence to: Dr. Yeliz Kllıc, Department of Anesthesiology and Reanimation, Faculty of Medicine, Osmangazi University, Eskişehir, Turkey

E-mail: yeliz_kilic3@hotmail.com

Received: October 20, 2020; Revised: November 27, 2020;

Accepted: November 27, 2020

DOI: https://doi.org/10.29271/jcpsp.2020.12.1256
To date, several therapeutic modalities such as prophylactic intravenous fluid replacement and vasopressor administration have been proposed to prevent postspinal hypotension. However, most of those have limited effectiveness, and are associated with potential hazardous consequences especially in patients with underlying cardiac and pulmonary diseases. ${ }^{7,8}$ Thus, accurate assessment of intravascular volume before anesthesia is of great importance for preventing or minimising such hypotensive events that may lead to serious problems.

Although central catheterisation is quite effective to predict the intravascular volume status, invasiveness, time-consuming property, and potential related complications limit its use, particularly in elective, short, or uncomplicated operations. Therefore, noninvasive and simple methods such as sonographic evaluation of the inferior vena cava (IVC) and internal jugular vein (IJV) have gained popularity in recent years. ${ }^{9,10}$ In the literature, there were few clinical studies investigated the effectiveness of sonographic measurements of IVCin the prediction of intravascular volume before spinal anesthesia. ${ }^{1,3,11}$ To the best of authors' knowledge, there is no study examining the effectiveness of IJV ultrasonography in predicting postspinal hypotension. This study aimed to investigate whether the sono- 
graphic measurements of IJV is an effective and useful diagnostic tool to predict postspinal hypotension in adult patients.

\section{METHODOLOGY}

Patients who underwent elective surgery under spinal anesthesia at Osmangazi University Hospital between January and September 2020 were enrolled in this prospective observational study (ClinicalTrials.gov Identifier: NCT04486612). The study was carried out in accordance with Helsinki Declaration after Ethics Committee's approval.

Informed consents were obtained from all participants before the procedure. Patients' age, gender, body mass index (BMI), American Society of Anesthesiologist (ASA) physical status, procedural data, anesthetic techniques, and perioperative complications related to anestheisa were recorded.

Patients aged between 18 and 65 years, with ASA score 1 or 2, who underwent elective surgery under spinal anesthesia in the supine position, were included in the study. Exclusion criteria were refusal to participate in the study, ASA physical status 3 and above, significant hepatic, renal, cardiovasculary, or respiratory disease, left ventricular ejection fraction less than $40 \%$, pregnancy, obesity $\left(\mathrm{BMI} \geq 30 \mathrm{Kg} / \mathrm{m}^{2}\right)$, emergency cases, surgical position other than supine position, use of angiotensin converting enzyme inhibitors, failure to perform spinal anaesthesia or need of additional sedoanalgesia, a baseline arterial systolic blood pressure (SBP) $<90 \mathrm{mmHg}$ or mean arterial pressure (MAP) $<70 \mathrm{mmHg}$, and allergy to the study medications.

All of the patients were fasted for at least eight hours before the operation, and did not have any premedication. In the operating room, standard monitoring included noninvasive blood pressure, five-lead electrocardiogram, and pulse oximetry. The measurements of baseline systolic (SBP), diastolic (DBP) and mean arterial blood (MAP) pressures, and heart rate (HR) were recorded. Subsequently, ultrasonographic examination was performed. An appropriate IV cannula was inserted in a peripheral vein, and an infusion of isotonic $\mathrm{NaCl}$ $(0.9 \%)$ solution was given at a rate of $10 \mathrm{ml} / \mathrm{kg} / \mathrm{h}$ immediately after spinal block.

Asecond anesthesiologist, whowas blinded to sonographicmeasurements, performed the spinal anesthesia and monitored the patient during surgery. Spinal (subarachnoidal) anesthesia was performed at L3-4 or L4-5 intervertebral space while the patient was in the sitting position. A 25 gauge spinal needle was used to administer spinal blockage at the level of toracal 8-10 with a dose of $10-12.5 \mathrm{mg}$ $0.5 \%$ hyperbaric bupivacaine. After intrathecal injection, the patients were immediately placed in the supine position, and remained in this position throughout the operation. Sensorial blockage level was assessed with a needle test. SBP, DBP, MAP, HR; and oxygen saturation were continuously monitored and recorded during the operation. Significant hypotension was defined as a MAP less than $65 \mathrm{mmHg}$ or more than $20 \%$ decrease compared to the baseline value. ${ }^{3}$ Patients were classified into two groups in accordance with this definition: patients with hypotension (hypotensive group) and those without hypotension (non-hypotensive group). Ephedrine $(5 \mathrm{mg}$ ) was administered when the OAB was below 60 $\mathrm{mmHg}$, and atropine $(0.5 \mathrm{mg})$ was administered when the HR fell below 50 perminute.
Ultrasonographic examinations were performed using a single ultrasound machine by a single anesthesiologist with an extensive experience in anesthesia practice. All patients were in supine position and having spontaneous breathing during the sonographic examinations. The curvilinear ultrasound probe (3.5-5 MHz) was placed horizontally to the right side of the middle level of the thyroid cartilage with minimal pressure, and clear transverse view of the right IJV was recorded for $15^{\text {th }}$ seconds (Figure 1A-B). The sonographic measurements of IJV were assessed using $\mathrm{M}$-mode imaging included maximum ( $\max$, at the end of expirium) and minimum ( $\mathrm{min}$, at the end of inspirium ) IJV diameters $(\mathrm{mm})$, max IJV area $\left(\mathrm{mm}^{2}\right)$, and collapsibility index $(\%)=[(\max I J V$ diameter - min IJV diameter $) /$ max IJV diameter $] \times$ 100. Based on the power analysis using the two-sample t-test, sample size of 22 patients per group was required to achive a power of $80 \%$ with a significant level of $5 \%$ for evaluating the differences between the groups with or without postspinal hypotension.

Data analysis was done using the statistical package for social science (SPSS 23.0 software, IL-Chicago-USA) standard version. Descriptive analyses were presented as number/percentage or mean \pm S.D and median (IQR: 25th percentile-75th percentile), respectively for categorical and continuous variables. Distribution of the variables was checked with Shapiro-Wilk t-test for normality. Independent sample t-test or Mann-Whitney U-test was used to evaluate the differences between the groups and the association between categorical variables was evaluated by using Chi-square test. In addition, receiver operating characteristic (ROC) curves were used to assess the diagnostic capability of IJV collabsibility index. A p value less than 0.05 was set as significance level.

\section{RESULTS}

Forty-seven patients with a median age of 38 (30-60) years were enrolled in the study. There were 16 (34\%) females and 31 $(66 \%)$ males. The mean BMI of the study population was 25.9 $\pm 2.8 \mathrm{Kgm}^{2}$ (19.4-29.9). During the preoperative evaluation, the majority of the patients $(32,68.1 \%)$ were classified as ASA 2; whereas, the remaining $(15,31.9 \%)$ had ASA 1 score. The mean fasting time was $12.6 \pm 1.8$ (8-16) hours. After spinal anesthesia, $22(46.8 \%)$ patients developed hypotension. The baseline patient characteristics and haemodynamic data were then compared between the hypotensive and non-hypotensive groups (Table I). The two groups were found to be similar in all baseline characteristics ( $p>0.05)$.

Four sonographic measurements of IJV were recorded: max and min diameters, max area, and collapsibility index. Thereafter, all these parameters were compared between the hypotensive and non-hypotensive groups (Table II). Max/min IJV diameters and max IJV area were similar between the two groups ( $p>0.05)$. Collapsibility index of IJV was found to be significantly lower in patients who developed postspinal hypotension compared to those without postspinal hypotension $(p=0.014)$. 
Table I: The comparison of baseline characteristics between the two groups.

\begin{tabular}{|c|c|c|c|}
\hline & $\begin{array}{l}\text { Hypotensive group } \\
(n=22)\end{array}$ & $\begin{array}{c}\text { Non-hypotensive group } \\
(\mathrm{n}=25)\end{array}$ & $\mathbf{p}$ \\
\hline Age (y) & $38(29.5-51.8)$ & $39(28-60)$ & 0.915 \\
\hline Gender (F/M) & $8(36.4 \% / 14(63.6 \%)$ & $8(32 \%) / 17(68 \%)$ & 0.753 \\
\hline BMI $\left(\mathrm{kg} / \mathrm{cm}^{2}\right)$ & $25.6 \pm 3.0(19.4-29.9)$ & $26.2 \pm 2.6(20.2-29.7)$ & 0.454 \\
\hline Fasting time $(\mathrm{h})$ & $12.7 \pm 1.5(10-15)$ & $12.4 \pm 2.0(8-16)$ & 0.584 \\
\hline Baseline SBP (mmHg) & $134.8 \pm 18.3(111-180)$ & $135.0 \pm 15.6(109-178)$ & 0.893 \\
\hline Baseline DBP(mmHg) & $75.3 \pm 12.1(56-100)$ & $79.0 \pm 12.2(58-97)$ & 0.357 \\
\hline Baseline MAP $(\mathrm{mmHg})$ & $97.8 \pm 13.5(78-126)$ & $100.7 \pm 12.0(77-129)$ & 0.448 \\
\hline Baseline HR (bpm) & $80.7 \pm 12.2(54-110)$ & $83.5 \pm 10.9(59-105)$ & 0.335 \\
\hline ASA (ASA1/ASA2) & $6(27.3) / 16(72.7 \%)$ & $9(36 \%) / 16(64 \%)$ & 0.522 \\
\hline
\end{tabular}

Data are presented as $n$ (\%) for gender and ASA; median (IQR: 25th percentile-75th percentile) or mean $\pm S D$ (min-max) for non-normally and normally

distributed variables, respectively. y: year, F: female, M: male, kg: kilogram, cm: centimeter, h: hour.

Table II: Comparison of sonographic data between the two groups.

\begin{tabular}{|l|c|c|}
\hline & $\begin{array}{c}\text { Hypotensive group } \\
(\mathbf{n = 2 2 )}\end{array}$ & $\begin{array}{c}\text { Non-hypotensive group } \\
\text { (n=25) }\end{array}$ \\
\hline Max IJV diameter (mm) & $9.1(7.5-10.5)$ & $9.2(7.8-11.7)$ \\
\hline Min IJV diameter (mm) & $6.9(6-8.2)$ & $6.5(5.2-8.7)$ \\
\hline Max IJV area (mm $\left.{ }^{2}\right)$ & $87(77.5-124.5)$ & $102(74-145)$ \\
\hline Collapsibility index (\%) & $17(11.1-32.6)$ & 0.677 \\
\hline \multicolumn{2}{|l|}{ Data are presented as median (IQR: 25th percentile-75th percentile). IJV: internal jugular vein, mm: milimeter. } \\
\hline
\end{tabular}

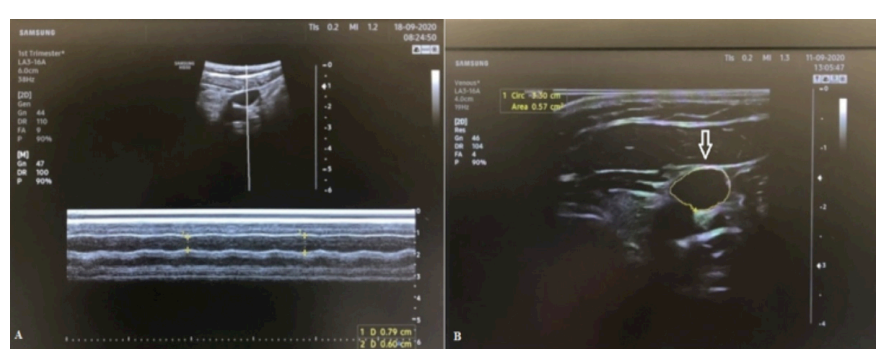

Figure 1: Sonographic view of: (A) max and min IJV diameters (at the end of expirium and inspirium, respectively); (B) max IJV area (arrow).

ROC curve analysis was used to evaluate the diagnostic ability of the collapsibility index of IJV in predicting the postspinal hypotension. IJV-Cl had a sensitivity of $64 \%$ and a specificity of $63.6 \%$ to predict the postspinal hypotension at a cut-off point of $22.6 \%$. The AUC was 0.709 (95\% confidence interval 0.56 to $0.86, p=0.014$, Figure 2 ).

\section{DISCUSSION}

Postspinal hypotension is common, and poses an important cause of concern for anesthesiologists, especially in high-risky patients such as the elderlies and those with underlying organ dysfunction. In this study, approximately half of the patients developed hypotension following spinal anesthesia, similar to the previous reports. ${ }^{12,13}$ Although not statistically significant, patients who developed hypotension were older than those without postspinal hypotension, consistent with general knowledge. ${ }^{2,3}$ It should be noted that excluding geriatrics and patients classified as ASA 3 and above may have affected this statistical result.

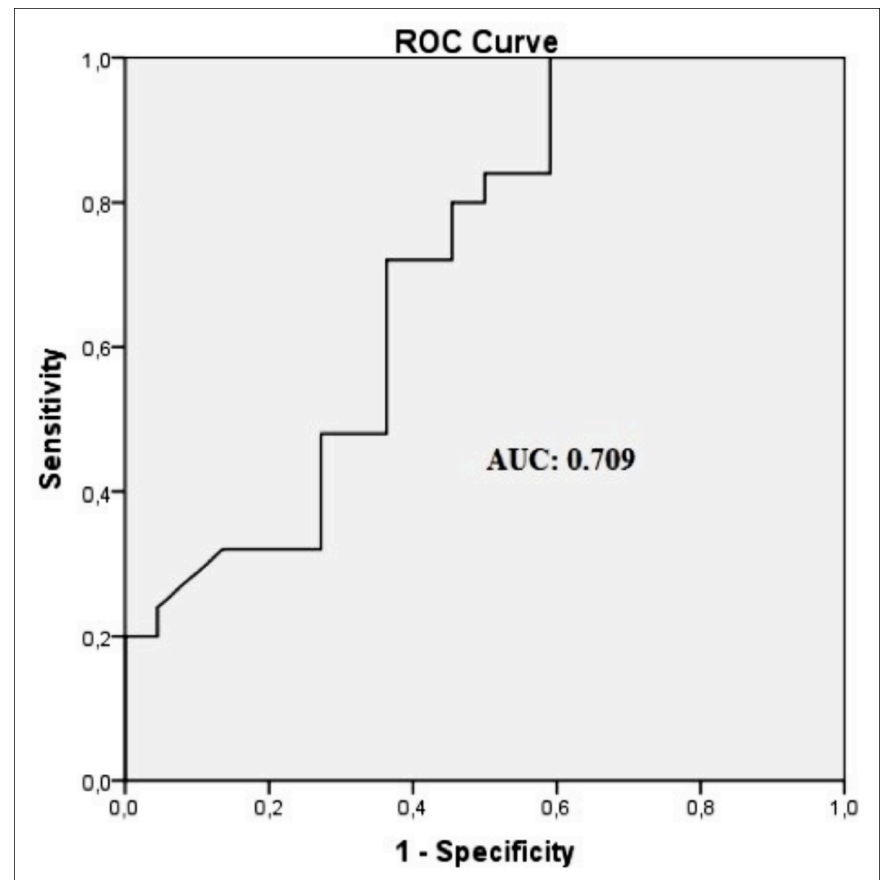

Figure 2: Receiver operating characteristic (ROC) curve analysis of the ability of IJV collapsibility index as predictor of postspinal hypotension. Area under curve (AUC) is $\mathbf{0 . 7 0 9 .}$

Today, real-time ultrasonography is increasingly used in various anesthesia applications such as regional blocks and intravascular volume assessment of intensive care patients. Recently, sonographic evaluation of great veins, such as IVC and IJV, in the assessment of intravascular volume status, has begun to gain popularity as a new application area. Considering that a significant number of the surgical patients may have low intravascular volume status, in other 
words latent hypovolemia, due to underlying chronic diseases, poor health status, or long fasting time, preanesthetic determination of this situation is of great importance to prevent potential related complications. Despite a universal definition, latent hypovolemia is a clinical entity described as a decrease in circulating blood volume without significant hemodynamic changes or organ dysfunctions. In addition, spinal anesthesia-induced sympathetic denervation causes reduction in systemic vascular resistance, which leads to relative hypovolemia. ${ }^{14-16}$

In the literature, there are limited number of studies investigating the use of ultrasonography in predicting postspinal hypotension. ${ }^{1,3,11,15}$ In the study by Singh et al., the authors examined the possible relationship between sonographic IVC measurements and postspinal hypotension, and found no positive correlation. The fact that the mentioned study was only conducted on young pregnant patients, who underwent cesarean section, can be considered as an important limitation for the generalisation of the statistical results. Contrary to that study, Salama et al. found in their work that IVC collapsibility index and IVC: aorta index were reliable predictors for the occurrence of postspinal anesthesia hypotension. ${ }^{3}$

The present study, on the other hand, focused on the determination of IJV sonographic examination in prediction of postspinal hypotension. Compared to IVC imaging, sonographic evaluation of IJV is easier and time-saving. Additionally, IVC imaging cannot be possible in a group of patients, due to abdominal gas shadow and obesity. Moreover, anesthesiologists are more familiar to IJV anatomy as they frequently perform ultrasound-guided central IJV catheterization in their routine practice. In the previous studies, various sonographic IJV measurements were determined as good predictors of fluid responsiveness, central venous pressure, or intravascular volume status. ${ }^{9,17,18}$ In a study conducted on both ventilated and non-ventilated patients, minimum and maximum IJV diameters revealed moderate correlation with central venous pressure. ${ }^{17}$ The study by Hossein-Nejad et al. ${ }^{19}$ also showed that sonographic IJV/CCA ratio could be an alternative and noninvasive tool of central venous catheterization in evaluating the hemodynamic status of critically ill patients. In another study, IJV aspect ratio less than 0.75 was found a reliable predictor of central venous pressure in spontaneously breathing ICU patients. ${ }^{20}$

In the literature, there is no study which investigated the use of IJV ultrasonography in predicting postspinal hypotension. However, sonographic IJV measurements in prediction of hypotension after general anesthesia induction was studied in one study by Okamura et al. ${ }^{21}$ In that study, the authors investigated the efficacy of preanesthetic $\mathrm{min} / \mathrm{max}$ diameters and area of IJV, and their changes with posture, in both supine and Trendelenburg positions. They found that only IJV area in the Trendelenburg position was an independent predictor for postinduction hypotension. IJV area was also found in another study as a sensitive parameter in predicting a central venous pressure under $5 \mathrm{mmHg} .{ }^{17}$ In this study, both diameters and area of IJV were not statistically different between hypotensive and non-hypotensive groups. Collapsibility index of IJV, on the other hand, was found to be a predictor of postspinal hypotension in the present study, despite moderate sensitivity and specifity rates. In recent years, IJV collapsibility index and other sonographic parameters are being increasingly used as an alternative to IVC in the measurement of central venous pressure. However, almost all of those studies have been conducted on acute, critical or ICU patients. ${ }^{22-24}$ The present study differed from those studies since it consisted of relatively healthy adults undergoing spinal anesthesia. In addition, very different cut-off values of IJV collapsibility index were reported in those works. ${ }^{22,25}$ This study is the first demonstrated the IJV collapsibility index is a risk factor for postinduction hypotension, with a cut-off value of $22.6 \%$. Similar to this study, Jaremko et al. demonstrated that median IVC-CI was lower in hypotensive patients $(24 \%)$ than in non-hypotensive patients $(30 \%) .{ }^{15}$ Differences in study populations, anesthesia types, and surgical prosedures may have contributed to this wide variation of cut-off values obtained from previous studies.

The present study has several limitations. Firstly, the fact that it was a single-centre study which may limit the generalisability of the statistical results. A relatively small number of patient groups may be considered as second limitation, which makes it difficult to interpret subgroup findings. A multi-centre or large scale study may also be required to evaluate possible cut-off values of some IJV parameters. Third, the study population consisted of participants with ASA1-2 scores which pointed out relatively good health status. Therefore, the results cannot be generalised to other patients groups such as pediatrics, geriatrics, or those with poorer health status. However, its prospective nature and standard anesthetic protocols were the strengths of this study. In addition, this is the first study which evaluated the diagnostic value of sonographic IJV measurements in the prediction of postspinal hypotension.

\section{CONCLUSION}

Despite the moderate sensitivity and specifity rates, collapsibility index of IJV can be used to be an alternative predictor of postspinal hypotension, with a cut-off value of $22.6 \%$.

\section{ETHICAL APPROVAL:}

This research was carried out after obtaining permission from the Ethics Committee of the Osmangazi University, Eskişehir, Turkey (Permit No./date:43/10.12.2019).

\section{PATIENTS' CONSENT:}

Consents were obtained from all patients.

\section{CONFLICT OF INTEREST:}

Authors declared no conflict of interest. 


\section{AUTHORS' CONTRIBUTION:}

YK: Study conception and design, acquisition of data, analysis and interpretation of data, drafting of manuscript.

SSSB: Study conception and design, acquisition of data.

GEK: Study conception and design, analysis and interpretation of data.

MSG: Analysis and interpretation of data, drafting of manuscript.

\section{REFERENCES}

1. Mačiulienè A, Gelmanas A, Jaremko I, Tamošiūnas R, Smailys A, Macas A. Measurements of inferior vena cava diameter for prediction of hypotension and bradycardia during spinal anesthesia in spontaneously breathing patients during elective knee joint replacement surgery. Medicina (Kaunas) 2018; 54(3): 49. Doi: 10.3390/Medicina 54030049 .

2. Zhang J, Critchley LA. Inferior vena cava ultrasonography before general anesthesia can predict hypotension after induction. Anesthesiology 2016; 124(3): 580-9. Doi: 10.1097/ ALN.0000000000001002.

3. Salama ER, Elkashlan M. Preoperative ultrasonographic evaluation of inferior vena cava collapsibility index and caval aorta index as new predictors for hypotension after induction of spinal anaesthesia: A prospective observational study. Eur J Anaesthesiol 2019; 36(4): 297-302. Doi: 10.1097/ EJA.0000000000000956.

4. Walsh M, Devereaux PJ, Garg AX, Kurz A, Turan A, Rodseth $\mathrm{RN}$, et al. Relationship between intraoperative mean arterial pressure and clinical outcomes after noncardiac surgery: Toward an empirical definition of hypotension. Anesthesiology 2013; 119(3): 507-15. Doi: 10.1097/ ALN.0b013e3182a10e26.

5. Wesselink EM, Kappen TH, Torn HM, Slooter AJC, Van Klei WA. Intraoperative hypotension and the risk of postoperative adverse outcomes: A systematic review. $\mathrm{Br} J$ Anaesth 2018; 121(4): 706-21. Doi: 10.1016/J.Bja. 2018.04.036.

6. Gu WJ, Hou BL, Kwong JSW, Tian X, Qian Y, Cui Y, et al. Association between intraoperative hypotension and 30-day mortality, major adverse cardiac events, and acute kidney injury after non-cardiac surgery: A meta-analysis of cohort studies. Int J Cardiol 2018; 258: 68-73. Doi: 10.1016/J.ijcard.2018.01.137.

7. Kweon TD, Kim SY, Cho SA, Kim JH, Kang YR, Shin YS. Heart rate variability as a predictor of hypotension after spinal anesthesia in hypertensive patients. Korean J Anesthesiol 2013; 65(4): 317-21. Doi: 10.4097/Kjae.2013.65.4.317.

8. Khan MU, Memon AS, Ishaq M, Aqil M. Preload versus coload and vasopressor requirement for the prevention of spinal anesthesia induced hypotension in nonobstetric patients. J Coll Physicians Surg Pak 2015; 25(12): 851-5. PMID: 26691355.

9. Unluer EE, Kara PH. Ultrasonography of jugular vein as marker of hypovolemia in healthy volunteers. Am J Emerg Med 2013; 31(1): 173-7. Doi: 10.1016/J.Ajem.2012.07.003.

10. Kalantari K, Chang JN, Ronco C, Rosner MH. Assessment of intravascular volume status and volume responsiveness in critically ill patients. Kidney Int 2013; 83(6): 1017-28. Doi:
10.1038/Ki.2012.424.

11. Singh Y, Anand RK, Gupta S, Chowdhury SR, Maitra S, Baidya DK, et al. Role of IVC collapsibility index to predict post spinal hypotension in pregnant women undergoing caesarean section. An observational trial. Saudi J Anaesth 2019; 13(4): 312-7. Doi: 10.4103/Sja.SJA_27_19.

12. Klöhr S, Roth R, Hofmann T, Rossaint R, Heesen M. Definitions of hypotension after spinal anaesthesia for caesarean section: Literature search and application to parturients. Acta Anaesthesiol Scand 2010; 54(8): 909-21. Doi: 10.1111/J.1399-6576.2010.02239.X.

13. Park S. Prediction of hypotension in spinal anesthesia. Korean J Anesthesiol 2013; 65(4): 291-2. Doi: 10.4097/ Kjae.2013.65.4.291.

14. Jor O, Maca J, Koutna J, Gemrotova M, Vymazal T, Litschmannova $M$, et al. Hypotension after induction of general anesthesia: Occurrence, risk factors, and therapy. a prospective multicentre observational study. J Anesth 2018; 32(5): 673-80. Doi: 10.1007/S00540-018-2532-6.

15. Jaremko I, Mačiulienè A, Gelmanas A, Baranauskas T, Tamošiūnas $\mathrm{R}$, Smailys $\mathrm{A}$, et al. Can the inferior vena cava collapsibility index be useful in predicting hypotension during spinal anaesthesia in a spontaneously breathing patient? A mini fluid challenge. Acta Med Litu 2019; 26(1): 1-7. Doi: 10.6001/Actamedica.V26i1.3948.

16. Szabó M, Bozó A, Darvas K, Horváth A, Iványi ZD. Role of inferior vena cava collapsibility index in the prediction of hypotension associated with general anesthesia: an observational study. BMC Anesthesiol 2019; 19(1): 139. Doi: 10.1186/S12871-019-0809-4.

17. Avcil M, Kapci M, Dagli B, Omurlu IK, Ozluer E, Karaman K, et al. Comparision of ultrasound-based methods of jugular vein and inferior vena cava for estimating central venous pressure. Int J Clin Exp Med 2015; 8(7): 10586-94. PMID: 26379848; PMCID: PMC4565231.

18. Hilbert T, Ellerkmann RK, Klaschik S, Putensen C, Thudium $M$. The use of internal jugular vein ultrasonography to anticipate low or high central venous pressure during mechanical ventilation. J Emerg Med 2016; 50(4): 581-7. Doi: 10.1016/J.Jemermed.2015.11.033.

19. Hossein-Nejad H, Mohammadinejad P, Ahmadi F. Internal jugular vein/common carotid artery cross-sectional area ratio and central venous pressure. J Clin Ultrasound 2016; 44(5): 312-8. Doi: 10.1002/Jcu.22339.

20. Prekker ME, Scott NL, Hart D, Sprenkle MD, Leatherman JW. Point-of-care ultrasound to estimate central venous pressure: A comparison of three techniques. Crit Care Med 2013; 41(3): 833-41. Doi: 10.1097/CCM.0b013e31827 $466 \mathrm{~b} 7$.

21. Okamura K, Nomura T, Mizuno Y, Miyashita T, Goto T. Preanesthetic ultrasonographic assessment of the internal jugular vein for prediction of hypotension during the induction of general anesthesia. J Anesth 2019; 33(5): 612-9. Doi: 10.1007/S00540-019-02675-9.

22. Killu K, Coba V, Huang Y, Andrezejewski T, Dulchavsky S. Internal jugular vein collapsibility index associated with hypovolemia in the intensive care unit patients. Crit Ultrasound J 2010; 2(1): 13-7. Doi: 10.1007/S13089- 010-0034-3.

23. Jassim HM, Naushad VA, Khatib MY, Chandra P, Abuhmaira 
MM, Koya SH, et al. IJV collapsibility index vs IVc collapsibility index by point of care ultrasound for estimation of CVP: A comparative study with direct estimation of CVP. Open Access Emerg Med 2019; 11: 65-75. Doi: 10.2147/OAEM.S176175.

24. Parikh R, Spring M, Weinberg J, Reardon CC, Farber HW. Use of ultrasound-measured internal jugular vein collapsibility index to determine static intracardiac pressures in patients with presumed pulmonary hypertension. Ann Intensive Care 2019; 9(1): 124. Doi: 10.1186/S13613-019-0595-7.

25. Haliloğlu M, Bilgili B, Kararmaz A, Cinel i. The value of internal jugular vein collapsibility index in sepsis. Ulus Travma Acil Cerrahi Derg 2017; 23(4): 294-300. Doi: 10.5505/Tjtes.2016.04832. 